\title{
Observations on Arrhenius Degradation of Lithium-Ion Capacitors
}

\author{
Davis George Moye ${ }^{1 *}$, Pedro L. Moss ${ }^{2}$, Xujie Chen\#, Wanjun Cao ${ }^{\#, ~ S i m o n ~ F o o ~}{ }^{3}$ \\ ${ }^{1}$ Moye Consultants, 527 E. Park Ave, Tallahassee, FL, USA \\ ${ }^{2}$ Greencastle Sustainable Electrical Energy Systems, Tallahassee, FL, USA \\ ${ }^{3}$ Department of Electrical and Computer Engineering, Florida A\&M University and Florida State University, \\ Tallahassee, FL, USA \\ Email: ^moyeconsultantsreceipts@gmail.com
}

How to cite this paper: Moye, D.G., Moss, P.L., Chen, X.J., Cao, W.J. and Foo, S. (2020) Observations on Arrhenius Degradation of Lithium-Ion Capacitors. Materials Sciences and Applications, 11, 450-461. https://doi.org/10.4236/msa.2020.117031

Received: May 12, 2020

Accepted: July 14, 2020

Published: July 17, 2020

Copyright (c) 2020 by author(s) and Scientific Research Publishing Inc. This work is licensed under the Creative Commons Attribution International License (CC BY 4.0).

http://creativecommons.org/licenses/by/4.0/

\begin{abstract}
Earlier research determined that lithium-ion capacitor (LIC) cycle life degradation can be accelerated by elevated temperature. LIC cycle life degradation can be described by an Arrhenius equation. This study performed cycle life testing at a constant temperature but varied cycle current. The results were described by an Arrhenius equation relying upon the number of cycles and a constant, which was determined by cycle current. Using mathematical derivations and experimental results, the researchers quantified the effects of activation energy and temperature upon this constant. Because cell temperature is nearly constant during cycles, it was deduced that elevated cycle current decreases activation energy. This lower activation energy then accelerates degradation. Thus this research demonstrates that cycle current ages LICs through its effects on their activation energies.
\end{abstract}

\section{Keywords}

Arrhenius Equation, Cycle Life, Cycle Life Degradation, Lithium-Ion Capacitor, Butler-Volmer Equation, Activation Energy

\section{Introduction}

Experimental work has provided significant insight into lithium-ion capacitor (LIC) performance [1] [2] [3] [4] [5]. LICs couple anodic lithium ion intercalation, a key aspect of lithium-ion batteries (LIB) with cathodic adsorption, characteristic of electrochemical double layer capacitors (EDLC). This enables LICs to simultaneously exploit the Faradaic reaction characteristic of a LIB and the 
non-Faradaic reaction characteristic of an EDLC [4]. LICs are thus hybrids between traditional LIBs and traditional EDLCs. LICs often have high specific power $\left(10 \mathrm{~kW} \cdot \mathrm{kg}^{-1}\right)$ and long cycle life (e.g. 300,000 cycles in laboratory experiments at General Capacitor) but low specific energy (5 - $\left.10 \mathrm{Wh} \cdot \mathrm{kg}^{-1}\right)$ [3].

Cao and Zheng have studied how LICs' internal resistance decreases at high temperatures [3]. Solid electrolyte interphase (SEI) is a thin layer that forms on the anode's surface as lithium reacts with the electrolyte or impurities in the cell. This SEI is essential to performance, as it enables the passage of lithium ions between the electrolyte and the porous carbon material. However, SEI growth can become too thick and impede the intercalation of lithium-ions into the negative electrode. This is a common degradation mechanism, which often leads to failure [6] [7].

Past LIC modeling studies have focused upon the effects of ambient and operating temperature upon cycle life or upon LIC performance as part of a larger system. Omar et al. [8] developed a LIC model by measuring LIC charge and discharge performance under varied temperatures, currents, and states of charge, as well as EIS data at different states of charge. Results were applied to a FreedomCar battery ECM. Barcellona et al. modeled LIC performance using modified EDLC performance models [8] [9] [10].

Concerning LIC cycle life, Uno and Kukita [11] experimentally validated an Arrhenius model previously proposed by Uno and Tanaka [7] using commercial-off-the-shelf (COTS) LICs. Additionally, El Ghossein et al. have described cycle life's effects on capacitance loss and increased equivalent series resistance (ESR) in terms of a Langmuir adsorption coefficient, which describes changes in an electrochemical double layer induced by gassing [12]. All of these studies collect data on pre-made, existing COTS LICs but do not consider any variables describing the electrochemistry inside the LICs.

This study seeks to understand cycle current's effects on cycle life degradation in LICs. It will be shown that findings from this study agreed with results from earlier studies, validating the research method employed here, which is described in Section 3.1.

\section{Earlier Research}

As aforementioned, electrolyte breakdown is a primary cause of EDLC degradation. This degradation is conveniently represented by an Arrhenius equation, as follows

$$
k=A_{r} \mathrm{e}^{-\frac{E_{a}}{R T}}
$$

where $k$ is the chemical reaction rate per cycle, $A_{r}$ is a rate constant, $E_{a}$ is the activation energy, $R$ is the gas constant, and $T$ is the absolute temperature in Kelvin [13]. $E_{a}$ is not constant but has been observed in the range $10-50 \mathrm{~kJ} \cdot \mathrm{mol}^{-1}$ for LICs [13]. Assuming an arbitrary constant (b)

$$
D_{T}=k b
$$


and

$$
A_{D}=A b
$$

wherein $D_{T}$ is the degradation ratio and $A_{D}$ is the degradation constant, Equation (1) above can be rewritten as follows

$$
D_{T}=A_{D} \mathrm{e}^{-\frac{E_{a}}{R T}}
$$

where $0 \leq D_{T} \leq 1$ [13]. From this equation $D_{T}$ can be used to compute the degradation acceleration factor based upon $10^{\circ} \mathrm{C}$ of $T$.

$$
\alpha=\left(\frac{T-T_{r e f}}{10}\right) \sqrt{\frac{D_{T}}{D_{T_{r e f}}}}
$$

where $\alpha$ is the acceleration factor, $T$ is the ambient temperature, $D_{T_{r e f}}$ is a degradation factor at a given reference ambient temperature, and $T_{r e f}$ is the reference ambient temperature [13]. From this relationship the per cent capacitance after degradation $\left(C_{d}\right)$ can be computed

$$
C_{d}=100-d_{T_{r e f}} \alpha\left(\frac{T-T_{r e f}}{10}\right) \sqrt{t}
$$

where $d_{T_{r e f}}$ is a degradation rate constant at $T_{\text {ref }}$ and $t$ is the time in number of cycles [13]. Uno et al. found that $D_{T}$ decreases over time and, consequently, the change in $C_{d}$ decreases as a function of time, creating an Arrhenius curve [7] [13].

Once $A_{D}, E_{a}$, and $T$ are known, a LIC's degradation can be predicted. This model relies upon experimentally-determined parameters. However, $A_{D}$ and $E_{a}$ are not the same for all manufacturers [11] and require either a previous test or detailed knowledge of the manufacturing process. An Arrenhius equation that accurately predicts the degradation of one LIC may not be accurate for another.

By contrast, another method computes capacitance degradation from the effects of temperature and voltage upon electrochemical double layer degradation as follows

$$
C_{d}=\left(\frac{c_{i}-\frac{a t}{1+v t}}{c_{i}}\right) \times 100
$$

where $c_{i}$ is the initial capacitance, $t$ is the cycle number, $a$ is the Langmuir adsorption coefficient as a function of temperature, and $v$ is the Langmuir adsorption coefficient as a function of voltage [12]. Langmuir adsorption coefficients balance a material's likelihood to enter a carbon material against its propensity to gas. This agrees with earlier studies indicating that dendrite formed by lithium precipitating out of carbon electrodes is a common degradation and failure mechanism in LICs [14]. Using Langmuir coefficients to study cycle life degradation may be a promising approach. In catastrophic failures both gassing and dendrites have been observed [15]. Also, gassing appears to be a sign of de- 
gradation in LICs [4] [16] during less dramatic failures. Most importantly, El Ghossein et al's model also accurately predicts a cycle life degradation curve, following an Arrhenius curve but does express degradation in the form of Equation (1).

At high temperatures LICs' internal resistance decreases [3]. At high temperatures a LIC initially has elevated capacity, but this soon diminishes and causes the LIC to age faster than it would while operating at room temperature. It is believed this phenomenon occurs because electrolyte breaks down and eventually forms impurities that impede the performance of the SEI [4]. This SEI is essential as a medium to enable passage of lithium ions from the electrolyte into the porous carbon material. However, if the SEI is impeded or contains impurities, the LIC's performance decreases [3]. Therefore, a LIC should degrade along an Arrhenius curve due to electrolyte breakdown. A key metric to validate this research is degradation along an Arrhenius relationship like those identified in [7] [12] [13].

Furthermore, the Butler-Volmer equation predicts an inverse relationship between cycle current and temperature as follows

$$
i_{d}=i_{0}\left(\mathrm{e}^{\frac{\alpha_{a} F n}{R T} \eta}-\mathrm{e}^{-\frac{\alpha_{c} F n}{R T} \eta}\right)
$$

where $i_{d}$ is current density, $i_{0}$ is exchange current density, $\alpha_{a}$ is the specific surface area of the anode electrode, $\alpha_{c}$ is the specific surface area of the cathode electrode, $n$ is the number of electrons per ion ( 1 for lithium), $\eta$ is the activation overpotential, $F$ is Faraday's constant, and $T$ is the absolute temperature of the electrochemical cell [1].

Because in an LIC the amount of charge stored at the negative electrode is orders of magnitude higher than the cathode [1], assume [17]

$$
\mathrm{e}^{-\frac{\alpha_{c} F n}{R T} \eta} \approx 1
$$

Therefore

$$
i_{d}=i_{0} \mathrm{e}^{\frac{\alpha_{a} F n}{R T} \eta}-1
$$

This assumption has proven accurate in past studies approximating energy storage as a function of constituent component materials, design, and operating conditions [16] [17]. Because

$$
i_{d}=\frac{i}{a}
$$

where $i$ is current and $A$ is the surface area

$$
i \propto \frac{1}{T}
$$

The relationships given in Equation (4) and Equation (12) indicate that cells cycled at constant temperature but different cycle currents should also degrade according to an Arrhenius equation taking the basic form 


$$
D_{T}=A_{i} \mathrm{e}^{-k_{i}}
$$

wherein $D_{T}$ is a degradation ratio, $A_{i}$ is a degradation constant specific to $i$, and $k_{i}$ is a kinetic constant dependent upon current, wherein

$$
i \propto k_{i}
$$

However, Moye et al. recently found that although Equation (12) is accurate, temperature change during a charge cycle inside an LIC is small $(<1 \%)$, and temperature changes are mostly observed in the low current regimes where Faradaic energy storage reactions dominate, similar to a lithium-ion battery [16]. At $i_{d}$ values in excess of $250 \mathrm{~A} \cdot \mathrm{kg}^{-1}$ no temperature increase was observed. Thus elevated current does not necessarily elevate temperature, but, as established by Uno et al. and El Ghossein et al., it does degrade a LIC in a similar manner [12] [13].

The main objective of this study was to determine why variations in cycle current degrade a LIC similarly to variations in cell temperature, although current does not appreciably change temperature.

\section{Experimental Work}

\subsection{Experimental Setup}

A cycle life evaluation was performed upon a LCA200G1 LIC made by General Capacitor, shown in Figure 1. General Capacitor has provided the design parameters for this product, as shown in Table 1.

General Capacitor has collected cycle life data on its LCA200G1. The LCA200G1 was one of General Capacitor's established commercial products, so data was easily obtained. Using an established, commercial product eliminated much of the experimental variability often encountered in laboratory-made LICs. Much LCA200G1 evaluation data has been made public in earlier studies [16] [17] [18].

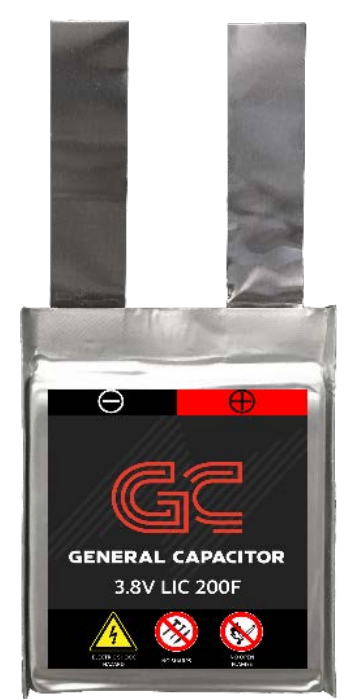

Figure 1. LCA200G1 LIC product made by General Capacitor. 
Table 1. Specifications of General Capacitor LCA200G1, flagship nominal 200F product made by General Capacitor. These parameters were verified by the authors, who were General Capacitor employees at the time, in accordance with normal company quality assurance processes. This product has been used as a reference in several earlier studies [16] [17] [18].

\begin{tabular}{|c|c|}
\hline Parameter & Value \\
\hline Lithium Source & Foil Strips \\
\hline Positive Electrode Active Material & Activated Carbon \\
\hline Positive Electrode Active Layer Thickness & $100 \mu \mathrm{m}$ \\
\hline Double-Sided Positive Electrodes & 7 \\
\hline Negative Electrode Active Material & Hard Carbon \\
\hline Negative Electrode Active Layer Thickness & $90-95 \mu \mathrm{m}$ \\
\hline Double-Sided Negative Electrodes & 6 \\
\hline Single-Sided Negative Electrodes & 2 \\
\hline Negative Electrode Lithium Loading & $8.81 \%$ \\
\hline Negative Electrode Porosity & $44.16 \%$ \\
\hline Positive Electrode : Negative Electrode Mass Ratio & 0.678 \\
\hline Lithium Source & Foil Strips \\
\hline Positive Electrode Active Material & Activated Carbon \\
\hline Positive Electrode Active Layer Thickness & $100 \mu \mathrm{m}$ \\
\hline Double-Sided Positive Electrodes & 7 \\
\hline
\end{tabular}

\subsection{Experimental Method}

Cycle life data was acquired at 4A and 5A for General Capacitor LCA200G1 LICs made in accordance with Table 1 . Testing conditions could not be perfectly controlled. The temperature in the testing room averaged $29^{\circ} \mathrm{C}-33^{\circ} \mathrm{C}$ and was subject to variations during several power outages and maintenance on nearby equipment. Recent studies have noted changes in LIC energy storage as a function of temperature [2] [4]. Consequently, the data showed many small perturbations, including diurnal variation.

To correct these perturbations a foil was applied, averaging 9 data points before and 9 data points after each data point of interest, extending each datapoint over a 24 hour period. In order for the relationships identified in Equations (4) and (8) to hold valid under this experimental method, an Arrhenius relationship indicating degradation over subsequent cycles must take the form

$$
\frac{c_{i}-c_{f}}{c_{i}}=A_{D} \mathrm{e}^{-k_{t} t}
$$

where $A_{D}$ is a degradation constant, $k_{t}$ is a kinetic constant encompassing $E_{a}$, 
$R$, and $T$, and $t$ is the cycle number. Although Equation (15) does not use Langmuir adsorption coefficients, like Equation (7) it does depend upon an exponential governed by $t . \quad c_{f}$ is the final capacitance of the LIC after degradation, related to $D_{T}$ by

$$
D_{T}=\frac{c_{i}-c_{f}}{t}
$$

where $c_{i}$ is the initial capacitance of the LIC [7]. Notice that in order to agree with Equation (4), Equation (15) must obey Equation (12). Combining Equation (4) with Equation (16) indicates

$$
c_{f}=c_{i}-t A_{D} \mathrm{e}^{-\frac{E_{a}}{R T}}
$$

Combining Equation (15) with Equation (17) indicates

$$
A_{D} \mathrm{e}^{-k_{t} t}=c_{i}-t A_{D} \mathrm{e}^{-\frac{E_{a}}{R T}}
$$

At $t=1$ cycle Equation (18) simplifies to

$$
A_{D} \mathrm{e}^{-k_{t}}=c_{i}-A_{D} \mathrm{e}^{-\frac{E_{a}}{R T}}
$$

If $A_{D}$ is close in value to $c_{i}$, then

$$
k_{t}=-\ln \left(1-\mathrm{e}^{-\frac{E_{a}}{R T}}\right)
$$

and

$$
E_{a}=-R T \ln \left(1-\mathrm{e}^{-k_{t}}\right)
$$

\section{Results}

Trend lines were extrapolated from the foiled data, as shown in Figure 2. The trendlines revealed the following Arrhenius relationships, which meet the form of Equation (15), where capacitance (c) is expressed as a percentage

$$
c=0.97 \mathrm{e}^{-7 \times 10^{-7} t}
$$

for $4 \mathrm{~A}$ cycles, and

$$
c=0.97 \mathrm{e}^{-1 \times 10^{-6 t}}
$$

For $5 \mathrm{~A}$ cycles. $A_{D}$ is a constant $0.97 . k_{t}$ is $7 \times 10^{-7}$ for $4 \mathrm{~A}$ cycles and $1 \times 10^{-6}$ for $5 \mathrm{~A}$ cycles. For $5 \mathrm{~A}$ cycles, assuming cell failure when $c_{f}$ is $80 \%$ of $c_{i}$, Equation (21) indicates 275,263 cycles. The only changing input variable is cycle current. $A_{D}$ remains a constant, as it should for two identical LICs with identical chemical and physical properties. Thus Equations (15) and (17) are met.

Notice that for both relationships

$$
i \propto k_{t}
$$

meeting the requirements of Equation (14). Solving Equation (21) for $E_{a}$ using the $k$ value given in Equation (22) and the nearly constant average ambient temperature of $31^{\circ} \mathrm{C}(304 \mathrm{~K})$ yields $E_{a}=30.4 \mathrm{~kJ} \cdot \mathrm{mol}^{-1}$. Likewise, solving Equation (21) for $E_{a}$ using the $k$ value given in Equation (23) yields $E_{a}=35.8 \mathrm{~kJ} \cdot \mathrm{mol}^{-1}$. 


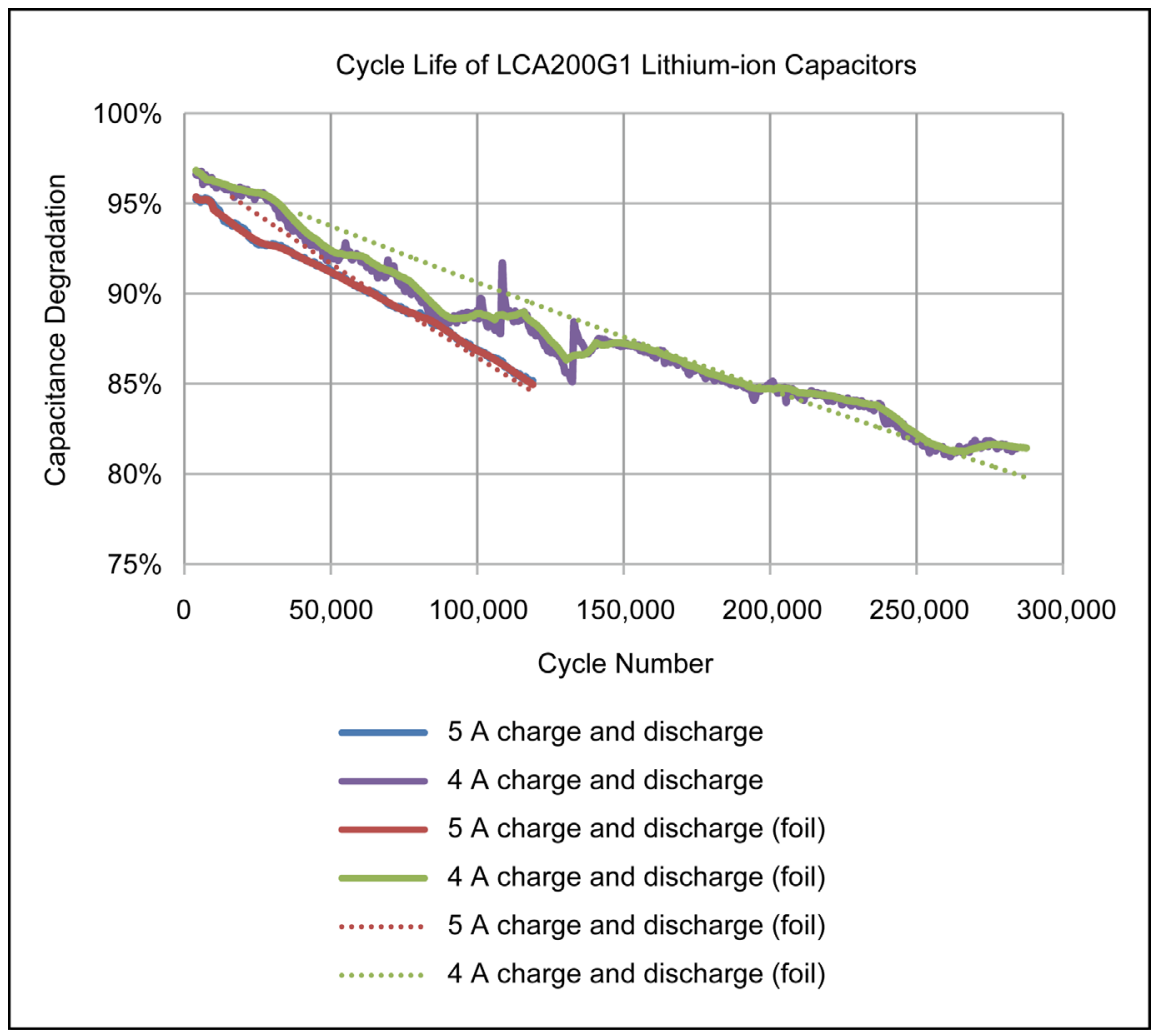

Figure 2. Cycle life graph of General Capacitor 200LCAG1s cycled at 5 A and 4 A. Minor perturbations can be attributed to ambient temperature as the room could not be perfectly held at a constant temperature but averaged $31^{\circ} \mathrm{C}$. Derived Arrhenius equations are shown. The only changing input variable is cycle current.

These values agree with $10-50 \mathrm{~kJ} \cdot \mathrm{mol}^{-1}$ reported by Uno and Tanaka. Thus elevating cycle current decreases $E_{a}$. In accordance with Equation (1), decreased $E_{a}$ accelerates degradation like increased $T$ does.

\section{Conclusions}

Prior to this study, it was well established that elevated temperatures accelerate LIC degradation. During this study, experimental LIC cycle life degradation at different cycle currents but constant ambient temperatures was approximated by Arrhenius equations as a function of the number of cycles. The Butler-Volmer equation indicates elevated cycle current may impact cell temperature and thereby degrade the LIC. However, other studies indicate that current does not induce much temperature increase in LICs. This study sought to understand why elevated current degrades a LIC without appreciably changing its temperature. Results indicate that cycle current decreases activation energy. These effects on activation energy degrade the LIC in the same manner as temperature.

Mathematically, these results agree with other, unrelated studies examing LIC degradation from the perspective of ambient temperature and the electrochemical double layer. Now that this study has demonstrated that dis/charge current affects activation energy as a degradation mechanism, future research should 
examine reasons for this phenomenon and eventually quantify the relationship between current and activation energy.

\section{Acknowledgements}

This research was performed using the resources of General Capacitor LLC and Moye Consultants LLC.

\section{Conflicts of Interest}

The authors declare no conflicts of interest regarding the publication of this paper.

\section{References}

[1] Zheng, J.P. (2003) The Limitations of Energy Density of Battery/Double-Layer Capacitor Asymmetric Cells. Journal of the Electrochemical Society, 150, A484-A492. https://doi.org/10.1149/1.1559067

[2] Cao, W., Li, Y., Fitch, B., Shih, J., Doung, T. and Zheng, J. (2014) Strategies to Optimize Lithium-Ion Supercapacitors Achieving High-Performance: Cathode Configurations, Lithium Loadings on Anode, and Types of Separator. Journal of Power Sources, 268, 841-847. https://doi.org/10.1016/j.jpowsour.2014.06.090

[3] Cao, W. and Zheng, J. (2012) Li-Ion Capacitors with Carbon Cathode and Hard Carbon/Stabilized Lithium Metal Powder Anode Electrodes. Journal of Power Sources, 213, 180-185. https://doi.org/10.1016/j.jpowsour.2012.04.033

[4] Boltersdorf, J., Delp, S.A., Yan, J., Cao, B., Zheng, J.P., Jow, T.R., et al. (2018) Electrochemical Performance of Lithium-Ion Capacitors Evaluated under High Temperature and High Voltage Stress Using Redox Stable Electrolytes and Additives. Journal of Power Sources, 373, 20-30. https://doi.org/10.1016/j.jpowsour.2017.10.084

[5] Cao, W.J., Shih, J., Zheng, J.P. and Doung, T. (2014) Development and Characterization of Li-Ion Capacitor Pouch Cells. Journal of Power Sources, 257, 388-393. https://doi.org/10.1016/j.jpowsour.2014.01.087

[6] Dahn, R., Zheng, T., Liu, Y. and Xue, J. (1995) Mechanisms for Lithium Insertion in Carbonaceous Materials. Science, 270, 590-593. https://doi.org/10.1126/science.270.5236.590

[7] Uno, M. and Tanaka, K. (2012) Accelerated Charge-Discharge Cycling Test and Cycle Life Prediction Model for Supercapacitors in Alternative Battery Applications. IEEE Transactions on Industrial Electronics, 59, 4704-4712. https://doi.org/10.1109/TIE.2011.2182018

[8] Omar, N., Ronsmans, J., Firozu, Y., Monem, M.A., Samba, A., Gualous, H., et al. (2013) Lithium-Ion Capacitor-Advanced Technology for Rechargeable Energy Storage Systems. World Electric Vehicle Symposium and Exhibition (EVS27), 17-20 November, Barcelona, 1-11. https://doi.org/10.1109/EVS.2013.6914718

[9] Barcellona, S., Ciccarelli, F., Iannuzzi, D. and Piegari, L. (2014) Modeling and Parameter Identification of Lithium-Ion Capacitor Modules. IEEE Transactions on Sustainable Energy, 5, 785-794. https://doi.org/10.1109/TSTE.2014.2301950

[10] Musolino, V., Piegari, L. and Tironi, E. (2013) New Full-Frequency-Range Supercapacitor Model with Easy Identification Procedure. IEEE Transactions on Industrial Electronics, 60, 112-120. https://doi.org/10.1109/TIE.2012.2187412 
[11] Barcellona, S. and Piegari, L. (2017) A Lithium-Ion Capacitor Model Working on a Wide Temperature Range. Journal of Power Sources. 342, 241-251. https://doi.org/10.1016/j.jpowsour.2016.12.055

[12] El Ghossein, N., Sari, A. and Venet, P. (2019) Lifetime Prediction of Lithium-Ion Capacitors Based on Accelerated Aging Tests. Batteries, 5, 28. https://doi.org/10.3390/batteries5010028

[13] Uno, M. and Kukita, A. (2016) Cycle Life Evaluation Based on Accelerated Aging Testing for Lithium-Ion Capacitors as Alternative to Rechargeable Batteries. IEEE Transactions on Industrial Electronics, 63, 1607-1617. https://doi.org/10.1109/TIE.2015.2504578

[14] Cao, W., Zheng, J., Adams, D., Doung, T. and Zheng, J.P. (2014) Comparative Study of the Power and Cycling Performance for Advanced Lithium-Ion Capacitors with Various Carbon. Journal of the Electrochemical Society, 161, A2087-A2092. https://doi.org/10.1149/2.0431414jes

[15] Bolufawi, O., Shellikeri, A. and Zheng, J.P. (2019) Lithium-Ion Capacitor Safety Testing for Commercial Application. Batteries, 5, 74.

https://doi.org/10.3390/batteries5040074

[16] Moye, D.G., Moss, P.L., Rajagopalan Kannan, D., Chen, X.J., Bolufawi, O., Cao, W.J. and Foo, S.Y. (2020) Improvements to Temperature, Warburg Impedance, and Voltage Computations for a Design-Based Predictive Model for Lithium-Ion Capacitors. Materials Science and Applications, 11, 347-369. https://doi.org/10.4236/msa.2020.116024

[17] Moye, D.G., Moss, P.L., Chen, X., Cao, W.J. and Foo, S.Y. (2019) A Design-Based Predictive Model for Lithium-Ion Capacitors. Journal of Power Sources, 433, Article ID: 226694. https://doi.org/10.1016/j.jpowsour.2019.226694

[18] Cao, W.J., Luo, J.F., Yan, J., Chen, X.J., Brandt, W., Warfield, M., Lewis, D., Yturriaga, S.R., Moye, D.G. and Zheng, J.P. (2017) High Performance Li-Ion Capacitor Laminate Cells Based on Hard Carbon/Lithium Stripes Negative Electrodes. Journal of the Electrochemical Society, 164, A93-A98.

https://doi.org/10.1149/2.0351702jes 


\section{Appendix}

\section{Nomenclature}

$A$ Langmuir adsorption coefficient as a function of temperature

A Ampere

$A \quad$ area

$A_{D} \quad$ degradation rate constant

$B \quad$ arbitrary constant

C Celsius

$C_{d} \quad$ per cent capacitance degradation

$c_{f} \quad$ final capacitance

$c_{i} \quad$ initial capacitance

COTS commercial off the shelf

$D_{T} \quad$ rate constant

$d_{T_{\text {ref }}} \quad$ degradation rate constant at $T_{\text {ref }}$

$D_{T_{\text {ref }}} \quad$ degradation factor at a given reference ambient temperature

$E_{a} \quad$ activation energy

ECM equivalent circuit model

EDLC electrochemical double layer capacitor

EIS electrochemical impedance spectroscopy

ESR equivalent series resistance

$F \quad$ Faraday's constant

g gram

i current

$i_{d} \quad$ current density

$i_{o} \quad$ exchange current density

k kilo

$k \quad$ Chemical reaction rate per cycle

K Kelvin

$k_{t} \quad$ kinetic constant relating $E_{a}, R$, and $T$ to $t$

LIB lithium-ion battery

LIC lithium-ion capacitor

mol moles

$n$ number of electrons per ion

$R \quad$ universal gas constant

$R_{c t} \quad$ charge transfer resistance

$R_{s} \quad$ series resistance

$R_{W} \quad$ Warburg resistance

SEI solid electrolyte interphase

$t$ time in numbers of cycles

$T \quad$ cell temperature

$T_{\text {ref }} \quad$ reference ambient temperature

$V \quad$ Langmuir adsorption coefficient 
Wh watt-hour

$\alpha \quad$ acceleration factor

$\alpha_{a} \quad$ anode specific surface area

$\alpha_{c} \quad$ cathode specific surface area

$\eta \quad$ activation overpotential 